\title{
Toward an Anti-Maleficent Research Agenda
}

\author{
Hope Ferdowsian $^{1}$, Agustin Fuentes ${ }^{2}$, L. Syd M Johnson ${ }^{3}$, Barbara J. King ${ }^{4}$ and Jessica Pierce 5 \\ ${ }^{1}$ Department of Medicine, University of New Mexico School of Medicine, Albuquerque, New Mexico 87106, USA \\ ${ }^{2}$ Department of Anthropology, Princeton University, Princeton, New Jersey 08544, USA \\ ${ }^{3}$ Center for Bioethics and Humanities, Upstate Medical University, Syracuse, New York 13210, USA \\ ${ }^{4}$ Department of Anthropology, William \& Mary, Williamsburg, Virginia 23187, USA \\ ${ }^{5}$ Center for Bioethics and Humanities, University of Colorado Anschutz Medical Campus, Aurora, Colorado 80045, USA \\ ${ }^{\star}$ Corresponding author: Email. hferdowsian@salud.unm.edu
}

\begin{abstract}
Important advances in biomedical and behavioral research ethics have occurred over the past few decades, many of them centered on identifying and eliminating significant harms to human subjects of research. Comprehensive attention has not been paid to the totality of harms experienced by animal subjects, although scientific and moral progress require explicit appraisal of these harms. Science is a public good and the prioritizing within, conduct of, generation of, and application of research must soundly address questions about which research is morally defensible and valuable enough to support through funding, publication, tenure, and promotion. Likewise, educational pathways of re-imagined science are critical.
\end{abstract}

Keywords: research ethics; animal subjects; morally defensible research

The past six decades have marked a significant revolution in research ethics in the United States. Following a number of research scandals, as described in Henry Beecher's influential 1966 New England Journal of Medicine paper, ${ }^{1}$ the National Commission for the Protection of Human Subjects of Biomedical and Behavioral Research published the Belmont Report, which established foundational ethical principles to guide research involving human subjects. ${ }^{2}$ One could describe their efforts to improve human research ethics as "antimaleficent"-focused on actively identifying and reducing actual and potential harms-within a broad framework of justice or fairness.

The current treatment of animals within the context of research suffers from some of the same problems that Beecher identified within human research. ${ }^{3}$ Despite significant advances over the past six decades in knowledge about the cognitive and emotional lives of animals, no effort to eliminate serious harms of research or to apply a thoroughgoing principle of justice, similar to that of the Belmont Report, has yet been advanced for animals. ${ }^{4}$

There is an urgent need for scientists and policymakers to acknowledge the importance of antimaleficence within the context of human and animal research. Progress requires an ethically rigorous evaluation of animal research, including a full articulation of the harms that animals experience during their use in research, and a reimagining of the financial, legislative, and social structures that perpetuate and reward harmful research.

\footnotetext{
(C) The Author(s), 2022. Published by Cambridge University Press. This is an Open Access article, distributed under the terms of the Creative Commons Attribution licence (http://creativecommons.org/licenses/by/4.0/), which permits unrestricted re-use, distribution, and reproduction in any medium, provided the original work is properly cited.
} 


\section{Evidence of Harm to Animals Used in Research}

\section{Physical and Physiological Harm}

As research targets, vertebrate animals are exposed to acute and chronic pain, including somatic or visceral nociceptive pain and central or peripheral neuropathic pain. Similarly, a growing body of research confirms that, despite their neuroanatomical differences from vertebrates, invertebrate animals also demonstrate coordinated responses to painful stimuli. ${ }^{5}$

Animals can also experience "sickness behavior" as a result of induced and by-product disease, which is characterized by lethargy, depression, anorexia, sleep disturbances, or increased pain sensitivity. ${ }^{6}$ Additionally, during development and adulthood, research environments can reshape neurobiological activity and connectivity, muscle and bone growth and structure, the functioning of sensory systems, and cardiovascular, endocrine, and digestive health.

Many animals' vulnerability to physiological harm can be partially explained by abnormalities in the hypothalamic-pituitary-adrenal axis, which have been described in animals who have been confined, restrained, or isolated, and after surgical procedures. ${ }^{7}$ Even low levels of trauma during early development can alter patterns and pathways of neurobiological and endocrine development such that adult functioning and capacities become constricted.

\section{Emotional and Psychological Harm}

Brain circuits and evolutionary histories prepare animals to experience and express a wide range of emotions. ${ }^{8}$ When animals in laboratories are made to undergo, and attempt to cope with, sustained experiences of anxiety, fear, sadness, grief, and anger, their bodies and minds are affected, re-shaped, and often damaged. This lasting harm alters their bodily and mental systems and stunts their capacity to feel and achieve healthy emotional lives.

Sustained adverse emotional states also increase the risk for psychological disorders. Attachment disorders, depression, complex anxiety disorders, posttraumatic disorders, and other persistent disorders of social behavior such as increased aggression and stereotypic behaviors are seen across many species of animals used in research. ${ }^{8}$

Adverse experiences early in life can especially affect development and the propensity for mental illness. Maternal-infant separation, confinement, social isolation, persistent fear and pain, and inadequate care can independently and cumulatively contribute to psychopathology in animals used in research.

\section{Sources of Harm in Biomedical and Behavioral Research}

\section{Captivity}

"Captivity effects" refer to the range of harmful physical, psychological, and physiological changes induced by confining captive conditions. These effects are similar in humans and animals, spanning across mammals, reptiles, amphibians, and fish. ${ }^{9}$

Research regulations often focus on the conditions of confinement (e.g., the size of cages and access to food and water) as a means of improving welfare. Mere improvements to captive conditions are insufficient to meet the totality of animals' needs; captivity itself is a central harm.

\section{Loss of Self-Determination and Bodily Sovereignty}

Many animals have evolved to govern their own lives, make decisions about how to satisfy their needs and avoid danger, and live within broader social and ecological networks. Animals used in research commonly experience violations of their bodily sovereignty. Even the technical aspects of research procedures and husbandry-including caging and the use of tranquilizers, anesthetics, paralytics, and other forms of physical and chemical force-commonly interfere with bodily sovereignty.

Like humans, some animals also have "higher order" needs, such as the need to exercise control over their lives. They may thrive only when they are able to form relationships with others and play, socialize, 
or otherwise act creatively. Self-determination is fundamental to satisfying higher order needs and it provides a necessary substrate for individuals to thrive. ${ }^{10}$

\section{Infliction of Pain, Injury, Disease, or Discomfort}

Animals used in research experience pain and discomfort as a result of a range of interventions including deprivation of or lack of control over access to food and water; extreme temperature exposure; physical restraint; deliberate infliction of injury, disability, and disease; medication side effects; incomplete medical or surgical care; and various methods of killing.

Invasive procedures also cause pain and discomfort through penetrating trauma (e.g., injections, surgical lacerations, or oral gavage), blunt force trauma (e.g., crush injuries involving the spinal cord or experimental trauma procedures), asphyxiation (e.g., near drowning with the "forced swim test"), burns (e.g., chemical or thermal burns, or electric shocks), restraint (e.g., head restraint with visual tracking experiments), and suspension (e.g., the "tail suspension test").

\section{Forced Sex, Coerced Reproduction, and Denial of Sexual Freedoms}

Many animals housed and used in research facilities are denied the opportunity to engage in a suite of reproductive behaviors including courtship, mating, gestation, and parental caregiving. Animals are also deprived of potential emotional experiences associated with sex and reproduction (e.g., sexual attraction, pleasure, and bonding). Heteronormative cage pairings and forced sex funnel animals into narrow behavioral patterns that prevent or constrain a diverse range of possibilities, including asexual behaviors and sexual behaviors with members of the same sex.

Other animals in captivity are forced to do nothing but reproduce, often through coercive human interventions. Female "breeders" are often forcibly inseminated, while male "semen donors" are subjected to electroejaculation (repeated electric "stimuli" to the penis), rectal massage, or masturbation of the male by a human.

Neonates are almost always taken from their mothers, imposing additional emotional wounds and physical deprivations.

\section{Deprivation of Life's Full Dimensions}

The brains of animals who dwell in rich, complex, and stimulating environments (e.g., their native habitats) have higher rates of new neuron-to-neuron connections, which means that vital brain activities like information-processing and memory capacity in these individuals increase. ${ }^{11}$

In sterile and boring conditions like laboratories, brain activity is dampened. The loss of opportunities for pleasurable sensory experiences and creative responses to them prevent animals from experiencing life's richness.

\section{Witnessing Harms}

When animals used in research suffer physical or emotional pain and distress, their conspecifics may witness these harms and experience trauma. Animals housed in pairs or small groups remain closely attuned to what happens together in that shared space; even when caged singly, individuals are often able to view and sense what happens to others around them because cages are in close proximity.

Because animals feel empathy of various types for each other, ${ }^{12}$ harms inflicted on animals multiply again and again, as the experience of one negatively affects many.

\section{Deception and Manipulation}

Practices that are often labeled "humane," such as positive reinforcement training of animal research subjects, are often manipulative, deceptive, and cause harm because they diminish control and enforce 
conversion to or compliance with the goals of researchers. Acquiescence in the absence of alternatives, such as the freedom to refuse or leave, does not constitute consent or assent.

\section{Being Killed}

Animals value their lives. Many animal species recognize and grieve death ${ }^{13}$ and will act to preserve their own lives and the lives of others. Being killed, and having one's life deliberately abbreviated, is a harm.

The manner in which animals used in research are killed can also cause pain and suffering. Methods for killing animals for research include decapitation by guillotine, cervical dislocation with mechanical force, asphyxiation, gassing, and lethal injection with barbiturates. Animals are also killed as part of experimental protocols, including toxicology tests designed to measure the lethal dose of a substance (e.g., LD50 and LD100 tests).

\section{Implications and Recommendations}

1) The research paradigm must become more ethical and just.

The time has come for research agendas to be shaped by social, economic, and cultural values that are inclusive and morally rigorous. Values guide science, and science cannot stand as an endeavor separate from history, social constructs, or current realities, including concrete evidence of agency and suffering in animals and well-recognized problems with the validity and reliability of research conducted on animal subjects. ${ }^{14}$

2) Federal funding needs to shift.

The priorities of funding agencies play a significant role in steering research. U.S. federal funding of scientific research gives little priority-and little money-to developing alternatives to animal research. The General Accountability Office report of 2019, "Animal Use in Research: Federal Agencies Should Assess and Report on Their Efforts to Develop and Promote Alternatives," contains details of the sparse funding for those alternatives. ${ }^{15}$

A genuine commitment to antimaleficence in research will require a significant financial commitment, in the form of earmarked funds for discovering and developing alternatives, and reduced funding for research with animals. Such a shift in priorities would be a radical departure from the status quo, and it is unlikely to happen without pressure from funding sources: Congress and taxpayers.

3) Academic journals and publishers must require more of authors.

Journals could incorporate a statement of adherence to an antimaleficent framework and to best practices, and a request that all submitted manuscripts do so as well. Submitted manuscripts that do not meet these expectations would need an addendum acknowledging the recognition and consideration of such criteria along with a specific justification in the addendum for how and why the guidelines were not met. Journal editors would then need to assess the validity of the rationale presented in the addendum and make appropriate editorial decisions. When researchers are found, postpublication, to have violated established rules, journals should retract papers for ethical breaches.

4) Reward structures, including tenure and promotion, need to emphasize and recognize ethics and innovation.

The reward structure in higher education, federal agencies, and other research institutions ought to reflect the value of research and teaching that develops, implements, uses, or advocates for the methods that follow the highest ethical standards-including an antimaleficent framework. Using this approach, tenure, promotion, financial reward, and protected time away from teaching and clinical care opportunities would recognize and reward ethical innovation. 
5) Education and training pathways need antimaleficent leadership and mentorship.

A key connection exists between the reward structure in higher education and research institutions and the training students receive in the sciences from elementary school through college and graduate education. What can be envisioned in research and teaching during one's career is influenced by one's experience as a student. If more students are to become researchers and educators who exhibit leadership in making scientific advances with ethical and just research, they must be exposed to and mentored in antimaleficent research methods.

\section{Conclusion}

The last century saw a dramatic shift in research with humans, toward more protection, and more just and ethical research. Science adapted. A comparable commitment to antimaleficence in research with animals would change the status quo, and it would change science as we know it. Much of the incidental and intentional harm that is inflicted on animals would no longer be permissible, and scientists would be called upon, as they were in generations past, to find new, better, and more ethical ways of engaging with science.

\section{Notes}

1. Beecher H. Ethics and clinical research. New England Journal of Medicine 1966;274:1354-60.

2. The Commission, Belmont Report: Ethical Principles and Guidelines for the Protection of Human Subjects of Research. Bethesda, MD: The Commission; 1978.

3. Ferdowsian H, Gluck, J. The ethical challenges of animal research: Honoring Henry Beecher's approach to moral problems. Cambridge Quarterly of Healthcare Ethics 2015;24:391-406.

4. Ferdowsian H, Johnson LSM, Johnson J, Fenton A, Shriver A, Gluck J. A Belmont report for animals? Cambridge Quarterly of Healthcare Ethics 2020;29:19-37.

5. Sneddon LU, Elwood RW, Adamo SA, Leach MC. Defining and assessing animal pain. Animal Behaviour 2014;97:201-12.

6. Hart BL, Hart LA. Sickness behavior in animals: Implications for health and wellness. In: Choe JC, ed. Encyclopedia of Animal Behavior. 2nd ed. Cambridge, MA: Academic Press; 2019, at 171-5.

7. Gregory NG. Physiology and Behavior of Animal Suffering. London, UK: Wiley; 2004.

8. Panksepp J. The basic emotional circuits of mammalian brains: Do animals have affective lives? Neuroscience \& Biobehavioural Reviews 2011;35:1791-804.

9. Bekoff M, Pierce J. The Animals' Agenda: Freedom, Compassion, and Coexistence in the Human Age. New York, NY: Penguin Random House; 2018.

10. Ryan RM, Deci EL. Self-Determination Theory: Basic Psychological Needs in Motivation, Development, and Wellness. 1st ed. New York, NY: The Guilford Press; 2017.

11. Markham JA, Greenough WT. Experience-driven brain plasticity: Beyond the synapse. Neuron Glia Biology 2004;1:351-63.

12. de Waal FBW, Preston SD. Mammalian empathy: Behavioral manifestations and neural basis. Nature Reviews Neuroscience 2017;18:498-509.

13. King BJ. How Animals Grieve. Chicago, IL: University of Chicago Press; 2013.

14. Bracken MB. Why animal studies are often poor predictors of human reactions to exposure. Journal of the Royal Society of Medicine 2009;102:120-2.

15. U.S. Government Accountability Office (GAO). Animal use in research: Federal agencies should assess and report on their efforts to develop and promote alternatives. (Publication GAO-19-629, GAO, 2019; https://www.gao.gov/assets/710/707338.pdf).

Cite this article: Ferdowsian, H. Fuentes, A. Johnson, L. S. M. King, B. J. and Pierce, J. 2022. Toward an Anti-Maleficent Research Agenda. Cambridge Quarterly of Healthcare Ethics 31: 54-58, doi:10.1017/S0963180121000487 